\title{
Genetic Analysis of $H 1$, the Structural Gene for Phase-1 Flagellin in Salmonella
}

\author{
By T. HORIGUCHI, S. YAMAGUCHI, K. YAO AND T. TAIRA \\ Department of Biology, School of Education, \\ Waseda University, Nishi-waseda, Tokyo I60, Japan \\ AND T. IINO \\ Laboratory of Genetics, Faculty of Science, \\ University of Tokyo, Hongo, Tokyo I I 3, Japan
}

(Received 7 April 1975; revised 3I May 1975)

\begin{abstract}
SUMMAR Y
For the mapping of $H I$, the structural gene for phase- I flagellin in Salmonella, spontaneous non-flagellate $H I$ mutants were isolated from a phase-I stable derivative, sJ925 $H_{I}-g_{1}, g_{2}, g_{3}, t$, of Salmonella abortusequi. First, mapping was carried out with the deletion mutants among them by $P_{22}$ phage-mediated transduction. Mutants of $f l a A I$ and $f l a L$, adjoining opposite sides of $H I$, were also included in the mapping. As the result, $H I$ was divided into 16 segments by 15 deletions. Mapping by recombination frequencies was then carried out using representative $H I$ mutants. Comparison of the two maps showed that I4 consecutive segments near $f l a L$ covered about $70 \%$ of the non-flagellate $H I$ mutational sites, although they were confined to a quarter of $H I$ in the recombination map. The other two segments were found to occupy the remaining three quarters of $H I$. By use of the deletion map, the sites of three phase-I curly and three $a \mathrm{hI}^{-}$mutations were determined. The curly mutational sites were mapped in the segment second from the flaAI side and the $a h^{-}$mutational sites in the segments near the flaL side. To ascertain approximate positions of the areas determining the phase-I antigen specificities, their arrangement relative to a curly mutational site, curly-2, and $H_{I}$-linked $f l a$ genes was examined by three-point crosses. From the results, all the antigenic specificity-determining areas examined were located between $f l a A I$ and curly-2 in the following order: flaAI- $g_{2}-g_{1}-g_{4}-\left(g_{3}, g_{5}, f, m, t\right)-c u r l y-2-f l a L$.
\end{abstract}

\section{INTRODUCTION}

In Salmonella, $\mathrm{HI}_{\mathrm{I}}$ and $\mathrm{H}_{2}$ were originally defined as the genes determining antigenic specificities of phase-I and phase-2 flagella, respectively (Lederberg \& Edwards, 1953). As it was later shown that the unit of flagellar antigen is the protein termed 'flagellin', which is the sole constituent of flagellar filaments, $\mathrm{HI}$ and $\mathrm{Hz}_{2}$ are now defined as the structural genes for flagellin of the respective phases (Iino, 1969). It is known that not only antigenic specificities but also the shape of the flagellar filaments are primarily determined by the $H$ genes (Iino, I962; Iino \& Mitani, 1966, 1967; Enomoto \& Iino, 1966).

Several papers have so far described the fine-structure analysis of the $H$ genes. By threepoint crosses, Joys \& Stocker (1969) attempted to determine the order of the sites of a curly mutation causing shortening of the wavelength of flagella and of mutations causing alterations in flagellar antigenic specificity, but could not reach a conclusive result. Yamaguchi \& Iino (1969) demonstrated intragenic recombination between $H r$ alleles 
specifying various forms of the g-complex antigens and mapped some antigenicallyimportant areas of the $H I$ gene in a linear array. Yamaguchi et al. (1972) reported a deletion map of $H I$ in which the gene was divided into four segments. To approach the problem of how the $H$ genes determine flagellar characters, more detailed genetic maps of these genes are indispensable.

In this study, a map of $H I$ was constructed with non-flagellate $H I$ mutants isolated from a phase-I stable derivative of Salmonella abortusequi. Further, by use of the map the locations of mutations responsible for phase-I curly flagella and of mutations which have been assumed to be in $a h I$, the region controlling the activity of $H I$, were determined. An attempt was also made to allocate antigenic specificity-determining areas within $H I$.

\section{METHODS}

Bacteria and phages. The characteristics and derivation of the Salmonella strains used are described in Table $\mathrm{I}$.

Phage $\chi$ M8 (Sasaki, 1962; Yamaguchi, 1968) is a host-range mutant of phage $\chi$. Phage $\chi$ attacks motile Salmonella organisms except for some specific serotypes including g-complex antigenic Salmonella (Meynell, 196I). The host-range of phage $\chi \mathrm{M} 8$ includes motile organisms with g-complex antigens.

Phage P22 was used as the mediator in transduction experiments.

Phage lysates were prepared by the soft-agar layer method and titrated on Salmonella strain SJ925.

Media. The compositions of nutrient broth, nutrient agar and semi-solid medium were described by Yamaguchi et al. (1972).

Isolation of non-flagellate mutants. Spontaneous non-flagellate mutants were isolated from a motile strain, SJ925, by the combined use of phage $\chi \mathrm{M} 8$ and semi-solid medium. The procedure was described by Yamaguchi et al. (1972).

Test for complementation and recombination. Complementation and recombination between pairs of non-flagellate mutants were examined by transduction. Production of trails (abortive transductants) and swarms (complete transductants) following transduction were used as the criteria for complementation and recombination, respectively (Yamaguchi et al. 1972).

For two-factor crosses, equal volumes of $\mathrm{P}_{22}$ phage suspension adjusted to $10^{10}$ infective units $/ \mathrm{ml}$ and bacterial suspension containing $10^{9}$ organisms $/ \mathrm{ml}$ were mixed. After 10 min adsorption, $0.1 \mathrm{ml}$ of the mixture was diluted 10 - or 50 -fold, and the whole quantity of the dilution was streaked on semi-solid medium plates. After overnight incubation at $37{ }^{\circ} \mathrm{C}$, the number of recombinants appearing as swarms was counted.

Recombination frequencies between the donor and the recipient mutational sites were expressed as percentages of the number of recombinants obtained when phage grown on the wild-type strain was used with the same recipients.

Identification of the antigenic specificity of flagella. Antigenic specificities of flagella were identified by slide agglutination with anti-factor(s) sera prepared according to Yamaguchi et al. (1969).

Description of bacterial strains. The mutant number, assigned from $210 I$ onwards for the non-flagellate $H I$ mutants derived from Salmonella strain SJ925, was also used to designate the corresponding mutational site.

For the description of the flagellar antigen type, the factor composition previously reported (Yamaguchi \& lino, 1969) was used, e.g. ' $g_{1}, g_{2}, g_{3}, t$ ' for that of strain SJ925, which 
Table r. Bacteria

\begin{tabular}{|c|c|c|}
\hline $\begin{array}{c}\text { Strain } \\
\text { no. }\end{array}$ & $\begin{array}{c}\text { Mutant } \\
\text { no. }\end{array}$ & Characteristics and derivation \\
\hline SJ925 & & $\begin{array}{l}\text { Motile phase-I stable derivative of Salmonella abortusequi sJ24I with } \\
H I-g_{1}, g_{2}, g_{3}, t \text { transduced from Salmonella sp. sJ6 (Yamaguchi et al. } \\
\text { I972) }\end{array}$ \\
\hline $\begin{array}{l}\text { SJI } 585 \\
\text { SJI } 727\end{array}$ & $\begin{array}{l}\mathrm{flaL204}_{2} \\
\mathrm{fla373}^{2}\end{array}$ & $\begin{array}{l}\text { Mutants of ss929, a derivative of sJ24I with } \mathrm{HI}_{I} \mathrm{~g}_{3}, g_{4}, f \text { transduced from } \\
\text { S. abony NCTCI } 729 \text { (Yamaguchi et al. I972) }\end{array}$ \\
\hline $\begin{array}{l}\text { SJW310 } \\
\text { SJW417 } \\
\text { SJi } 690\end{array}$ & $\begin{array}{l}\text { AaAII } 310 \\
\mathrm{HI}-\mathrm{IIO4} \\
\mathrm{AaL} 309\end{array}$ & Mutants of S. abortusequi ss24I $H I-a$ (Yamaguchi et al. 1972) \\
\hline SJ 1632 & flaAII25I & $\begin{array}{l}\text { Mutants of sJ930, a derivative of sJ24I, with } H I-g_{1}, g_{2}, g_{4}, g_{5}, m \text { transduced } \\
\text { from } S \text {. enteritidis NCTC4I96 }\end{array}$ \\
\hline $\begin{array}{l}\text { SJI } 891 \\
\text { SJI } 892 \\
\operatorname{SJI} 893\end{array}$ & $\begin{array}{l}H I-\text { curly-I } \\
H I \text {-curly-2 } \\
H I \text {-curly-3 }\end{array}$ & Phase-I curly mutants derived from ss929 $H I-g_{3}, g_{4}, f$ \\
\hline $\begin{array}{l}\text { sw1061 } \\
\text { sw629 } \\
\text { sw547 }\end{array}$ & $\begin{array}{l}a h I-I \\
a h I-2 \\
a h I-3\end{array}$ & O-H variants derived from $S$. typhimurium (Iino, 196I) \\
\hline
\end{tabular}

means that the flagellar antigen consists of factors $g_{1}, g_{2}, g_{3}$ and $t$. The chromosomal area determining the specificity of an antigenic factor was described by the italicized form of the symbol for the corresponding factor, e.g. $g_{1}, g_{2}, g_{3}$, etc. The italicized symbol was also used to designate the $H I$ allele, e.g. $H I-g_{1}, g_{2}, g_{3}, t$ for that of strain sJ925, which means that the $H I$ allele carries antigenic specificity-determining areas $g_{1}, g_{2}, g_{3}$ and $t$.

\section{RESULTS}

\section{Isolation and characterization of $\mathrm{HI}$, flaAI and flaL mutants}

Using phage $\chi \mathrm{M} 8$ as the selecting agent, 4247 spontaneous non-flagellate mutant clones were isolated from a phase-I stable strain, $\mathrm{SJ} 925 \mathrm{HI}-g_{1}, g_{2}, g_{3}, t$.

The non-flagellate phenotype is caused not only by mutation in $\mathrm{HI}$ but also in any of the $f l a$ genes. As it has been found that among the 15 known $f l a$ genes, $f l a A I$ and $f l a L$ adjoin opposite sides of $H I$ (Yamaguchi et al. 1972), mutants of flaAI and flaL as well as $H I$ were looked for among those isolated.

To identify $H I, f a A I$ and $f l a L$ mutants, all the non-flagellate clones obtained were subjected to complementation tests with three strains, SJW4I $7 \mathrm{HI}$-IIO4, SJW3 IO flaAII $3 \mathrm{IO}_{\mathrm{O}}$ and SJI $585 f_{1 a L 204}$, respectively. These indicator strains were used as donors in transduction experiments. As a result, 67 clones were identified as $H I$ mutants, I4 as $f l a A I$, IO2 as $f l a L$, and five as $H I$ flaL. Among them, 2 I $H I$, eight $f a A I$, I 6 flaL and all five $H I$ flaL mutants were stable, and the others revertible (Table 2).

Ten mutants were chosen at random from each of the three complementation groups and examined in all pairwise combinations for complementation. In all the inter-cistronic crosses, complementation was perfect, i.e. as many trails as those in the crosses with the wild-type donor were produced. In intra-cistronic crosses no complementation occurred except for some crosses between flaL mutants, in which fewer than $50 \%$ of the trails in the control crosses were produced. 
Table 2. Characteristics of $\mathrm{HI}$, flaAI, and flaL mutants isolated from Salmonella strain sJ925 and used for deletion mapping

Complementation
group

$H I$

flaAI
flaL

HI flaL

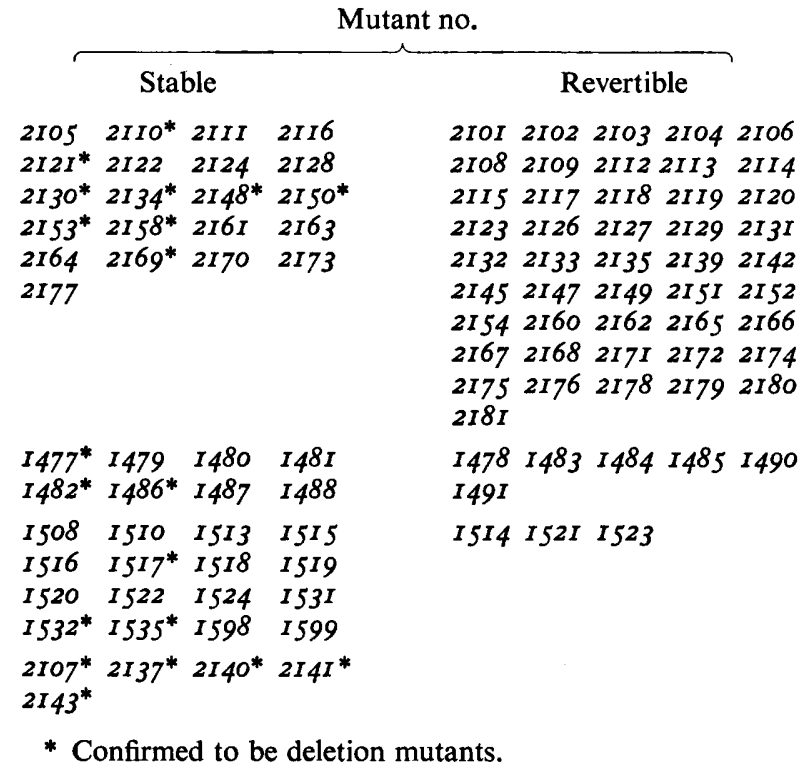

Deletion mapping of $\mathrm{HI}$ and its vicinity

Deletion mapping was achieved by the following two series of crosses. First, using the mutants listed in Table 2, transductions were carried out from each revertible mutant to all the stable ones. Stable mutants failing to produce swarms by transduction from more than two revertible mutants were regarded as deletion mutants. As a result, ten $H I$, three $f a A I$, three $f l a L$ and all five $H I$ flaL mutants were confirmed to be deletion mutants. Transductions were then carried out in all possible combinations among the deletion mutants. In these crosses, SJW3 10 flaAII 310 , SJI 585 flaL204 and SJI690 flaL309, whose mutations have been established to be deletions (Yamaguchi et al. 1972; Yamaguchi, unpublished), were also included. On the basis of their overlapping pattern, it was shown that all the $H I, f l a L$ and $H I$ flaL deletions were arranged in a contiguous sequence, and the four flaAI deletions in another contiguous sequence.

Combining the results of these two series of crosses, a deletion map (Fig. I) was constructed. $H I$ was divided into I 6 segments, $f l a A I$ into six segments and $f l a L$ into eight segments. In this map, stable mutations which were not confirmed to be deletions were included in the group of single site mutations. The orientation of $\mathrm{flaAI}$ segments with respect to $\mathrm{HI}$ was determined to be as shown in Fig. I by a three-point cross test between SJW478

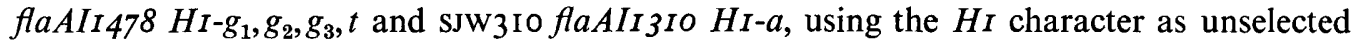
marker according to the method of Yamaguchi et al. (1972). Segments in the HI gene were given consecutive numbers from I to 16 running from the right-hand $(f a L)$ side to the left.

\section{Mapping by recombination frequencies}

In the deletion map (Fig. I) the segments were arbitrarily shown as of equal length. To know more precisely the distribution of the mutational sites, mapping by recombination frequencies in two-factor crosses was carried out. The mutational site of strain sJW510 

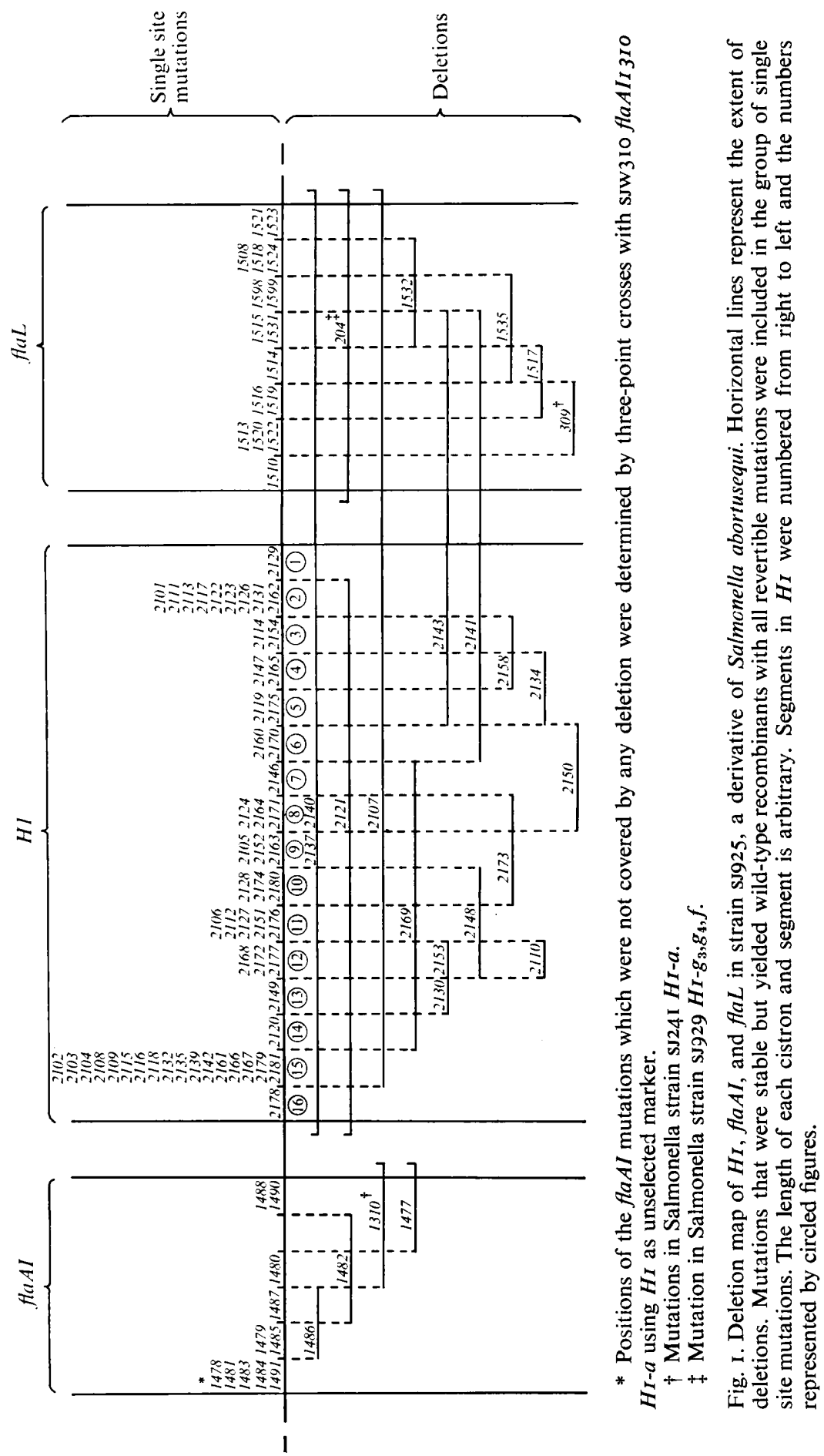
Table 3. Recombination frequencies between strain sJw5 10 flaLI 5 Io and non-flagellate Hr mutants

\begin{tabular}{|c|c|c|c|}
\hline \multirow[b]{3}{*}{$\begin{array}{c}\text { Recipient } \\
\text { (mutant no.) }\end{array}$} & \multirow{2}{*}{\multicolumn{2}{|c|}{$\begin{array}{l}\text { For details, see Methods. } \\
\text { No. of motile recombinants } \\
\text { in transduction from }\end{array}$}} & \multirow{3}{*}{$\begin{array}{l}\text { Recombination } \\
\text { frequency } \\
\text { (B/A, \%) }\end{array}$} \\
\hline & & & \\
\hline & $\begin{array}{l}\text { SJ925 (wild-type) } \\
\text { (A) }\end{array}$ & $\begin{array}{l}\text { SJW5 Io flaLI5Io } \\
\text { (B) }\end{array}$ & \\
\hline flaLi 5 Io & 1400 & 0 & 0 \\
\hline$H I-2 I 29$ & 1050 & I57 & 15.0 \\
\hline$H I-2 I 22$ & 990 & 161 & $16 \cdot 3$ \\
\hline$H I-2 I I I$ & 1240 & 206 & 16.6 \\
\hline$H I-2 I 49$ & 1040 & 184 & $17 \cdot 7$ \\
\hline$H I-2 I 2 O$ & I 100 & 22 I & $20 \cdot \mathrm{I}$ \\
\hline$H I-2 I I 6$ & 1220 & 250 & 20.5 \\
\hline$H I-216 I$ & 950 & 278 & $29 \cdot 3$ \\
\hline$H I-2 I 78$ & 1220 & 425 & $34 \cdot 8$ \\
\hline flaAl1490 & I 100 & 435 & $39 \cdot 3$ \\
\hline
\end{tabular}

flaLI5IO, which is located in the segment of flaL nearest to $H I$, was used as the standard site. Transductions were carried out from strain SJW5IO flaLI5IO to eight $\mathrm{HI}$ mutants chosen from various segments and strain SJW490 flaAII 490 whose mutational site is located in the segment of $f l a A I$ nearest to $H I$. The number of motile recombinants appearing as swarms on semi-solid medium was counted. As a control, transduction was carried out from the wild-type strain $\mathrm{sJ925}$ to each of the recipients. The relative recombination frequencies between flaLI5IO and the recipient mutational sites were calculated by taking the number of motile recombinants evoked in the respective control transductions as $100 \%$. From the data (Table 3) the map shown in the lower part of Fig. 2 was constructed. The mutational sites in $H I$ were distributed in the range 15.0 to $34.8 \%$. Their relative order fully accorded with that in the deletion map. The main thing disclosed by comparison of the recombination map with the deletion map is that mutational sites located at segments I to 14 in the latter were concentrated in a region comprising a quarter of the $H I$ gene, to its $f l a L$ side, in the former map. As shown in Fig. I, about $70 \%$ of the non-flagellate $H I$ mutational sites were mapped in segments I to I4. Thus the quarter at the flaL side of the $H I$ gene is considered to be 'hot' as regards the occurrence of spontaneous non-flagellate mutations compared with the other three quarters. It was shown that $H I-2 I I 6$ and $H I-2 I 6 I$, both of which were allocated to segment 15, mapped at positions 20.5 and 29.3 in the recombination map, respectively, indicating that segment 15 occupies most of the remaining three-quarters of the gene.

\section{Mapping of mutational sites responsible for curly flagella}

The genetic change of flagellar shape is due to mutation at sites in $\mathrm{HI}$ or $\mathrm{Hz}$; depending on which gene mutates, the mutant shape appears in either phase-I or phase-2 (Iino, 1969). The most commonly occurring flagellar-shape mutants are curly; their flagella are characterized by a wavelength about half the normal one.

Using $H I$ deletions, we attempted to locate the mutational sites of three phase-I curly mutants, SJI89I HI-curly-I, SJI892 HI-curly-2 and SJI893 HI-curly-3, in the deletion map. Transductions were carried out from the curly mutants to all the $H I$ deletion mutants.

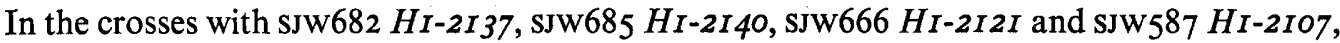
none of the curly mutants evoked swarms, while in other crosses they evoked swarms. 


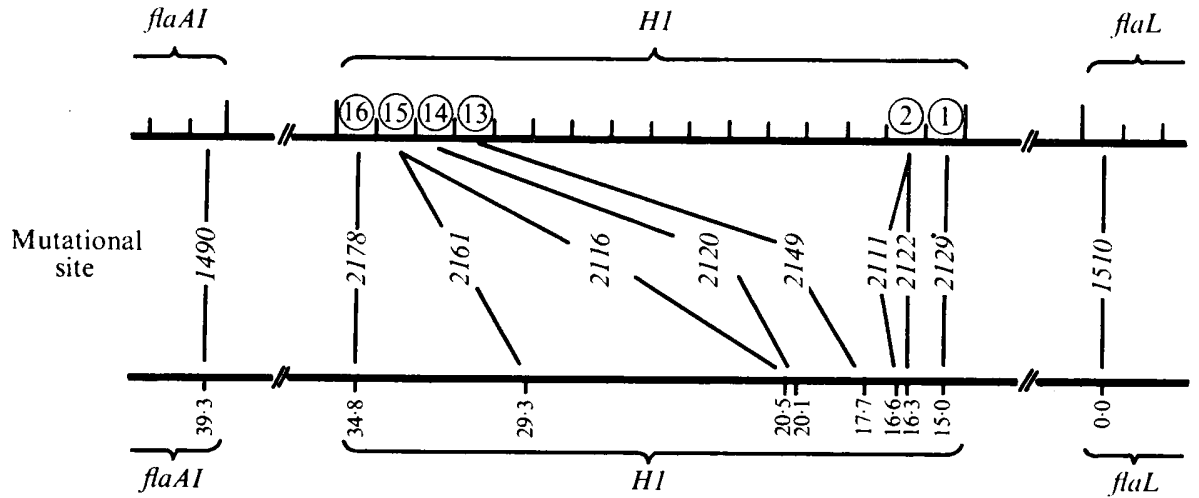

Fig. 2. Comparison between deletion and recombination maps of the flaAI-HI-flaL region. Above: Deletion map. Circled figures represent the segment numbers. Below: Recombination map. Figures below the horizontal line represent the distances from flaLI5IO, made proportional to recombination frequencies.



Fig. 3. Location of phase-I curly and $a h I^{-}$mutational sites in the $H I$ gene.

Consequently, the three curly mutational sites were allocated to segment 15 where the four deletions overlap (Fig. 3).

\section{Mapping of ahI $I^{-}$mutations}

$\mathrm{O}-\mathrm{H}$ variation in some diphasic Salmonella strains has been attributed to a mutant allele of $a h_{I}$ ( $H I$-activity controller) which is closely linked to but separable from antigen type determinants of the $H I$ gene (Iino, I96I, I969). The position of ahI relative to $H I$ has not yet been determined.

By the same method as used in the mapping of curly mutational sites, the mutational 
sites of three previously-reported $a h^{-}$mutants of Salmonella typhimurium, SWI06I ahI-I, Sw629 $a h r-2$ and Sw547 $a h r-3$, were mapped. As shown in Fig. 3, they were allocated to three different segments near $f a L$ within the $H I$ region.

\section{Mapping of antigenic specificity-determining areas}

The flagellar antigen is a complex composed of several antigenic factors differing in specificity; that is, one flagellin molecule carries several distinct antigenic determinants. By intragenic recombination between $H_{I}$ alleles specifying various kinds of g-complex antigens, the following arrangement of some antigenic specificity-determining areas in the $H I$ gene was disclosed (Yamaguchi \& Iino, I969):

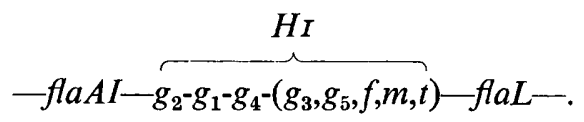

To combine this map with the present one, we attempted to determine the position of antigenic specificity-determining areas relative to a curly mutational site by the following three-point cross tests. Transductions were carried out from a curly mutant to $H I$-linked fla mutants derived from motile strains carrying different antigenic factor compositions, and hence different $H I$ alleles, from those of the donor; then motile recombinants were selected on semi-solid medium and their antigenic factor compositions were examined.

As the donor curly mutant, SJI892 $\mathrm{HI}$-curly-2, whose $\mathrm{HI}_{\mathrm{I}}$ allele is $\mathrm{HI}_{\mathrm{-}} \mathrm{g}_{3}, \mathrm{~g}_{4}, f$,curly, was used. Transduction was first carried out from the donor to a deletion mutant, SJI 726 fla373, whose deletion covers $f l a A I, A I I, A I I I, B, D, N, P, Q$ and $R$ and whose $H I$ allele is $H I-g_{1}, g_{2}, g_{3}, t$. Among 57 motile transductants obtained, two clones carried the donortype factor composition and 53 the recipient-type one, while the remaining two carried hybrid-type ones, that is , ' $\mathrm{g}_{1}, \mathrm{~g}_{3}, \mathrm{t}$ ' (hybrid-type $\mathrm{I}$ ) and ' $\mathrm{g}_{3}, \mathrm{~g}_{4}, \mathrm{t}$ ' (hybrid-type II), respectively (Table 4). Assuming that all the recombinants resulted from two crossovers, one in the region between $f l a 373$ and curly-2 and the other beyond fla373, the occurrence of the above four types of recombinants can be explained only when antigenic specificitydetermining areas are located as shown in Table $5 \mathrm{~A}$. That is, the donor-type clones are considered to have resulted from two crossovers in regions I and 5 in Table $5 \mathrm{~A}$, the recipienttype ones in regions I and 2, the hybrid-type-I clone in regions I and 3, and hybrid-type-II clone in regions $I$ and 4 . Therefore, the following order of antigenic specificity-determining areas relative to curly-2 and fla373 is inferred:

$$
-f l a 373-g_{2}-\left(g_{1}, g_{4}\right)-(f, t)-c u r l y-2-.
$$

As $g_{3}$ is common to both donor and resipient $H I$ alleles, its position cannot be inferred from this cross.

Transduction was next carried out from sJi892, the same donor as above, to sJI632 flaAII25I, whose $H_{I}$ allele is $H_{I-g_{1}}, g_{2}, g_{4}, g_{5}, m$. Among 104 motile transductants obtained, four carried the donor-type factor composition and Ioo carried the recipient-type one. The occurrence of these two types of transductants is explained as shown in Table $5 \mathrm{~B}$. That is, crossovers in regions $I$ and 3 resulted in the production of the donor-type recombinants and those in regions $I$ and 2 resulted in the production of the recipient-type ones. Thus, the order

$$
\text { - flaAII25I- }\left(g_{1}, g_{2}, g_{3}, g_{5}, f, m\right) \text {-curly-2- }
$$

is inferred. As $g_{4}$ is common to both donor and recipient $H I$ alleles, its position cannot be inferred from this cross. 
Table 4. Flagellar antigens of recombinants obtained in crosses between a curly mutant and fla mutants carrying different $\mathrm{HI}$ alleles

Transduction was carried out from the curly mutant to fla mutants.

\begin{tabular}{|c|c|c|c|c|c|}
\hline \multirow{2}{*}{\multicolumn{2}{|c|}{ Transduction }} & \multicolumn{4}{|c|}{ No. of recombinants } \\
\hline & & Donor- & Recipient- & Hybrid- & \\
\hline Donor & Recipient & type* & type* & type & Total \\
\hline$H I-g_{3}, g_{4}, f$, curly-2 & $\begin{array}{l}\text { SJI } 727 \text { fla } 373 \ddagger H I-g_{1}, g_{2}, g_{3}, t \\
\text { SJI632 flaAII } 25 I H I-g_{1}, g_{2}, g_{4}, g_{5}, m\end{array}$ & $\begin{array}{l}2 \\
4\end{array}$ & $\begin{array}{r}53 \\
100\end{array}$ & $\begin{array}{l}2 \\
0\end{array}$ & $\begin{array}{r}57 \\
104\end{array}$ \\
\hline
\end{tabular}

* Factor composition of the donor-type is ' $\mathrm{g}_{3}, \mathrm{~g}_{4}, \mathrm{f}$ ' in both crosses, and those of the recipient-types are ' $\mathrm{g}_{1}, \mathrm{~g}_{2}, \mathrm{~g}_{4}, \mathrm{t}$ ' in the upper cross and ' $\mathrm{g}_{1}, \mathrm{~g}_{2}, \mathrm{~g}_{4}, \mathrm{~g}_{5}, \mathrm{~m}$ ' in the lower one.

$\dagger$ Factor compositions of the two clones obtained in the upper cross are ' $\mathrm{g}_{1}, \mathrm{~g}_{3}, \mathrm{t}$ ' and ' $\mathrm{g}_{3}, \mathrm{~g}_{4}, \mathrm{t}$ ', respectively.

$\ddagger f l a 373$ is a deletion covering $f l a A I, A I I, A I I I, B, D, N, P, Q$, and $R$.

Table 5. Possible arrangement of antigenic specificity-determining areas in $\mathrm{HI}$ with respect to curly and fla mutational sites, that explains the occurrence of the recombinants shown in Table 4

Possible arrangement

(A) Transduction from SJI $892 \mathrm{HI}_{-} g_{3}, g_{4}, f$,curly-2

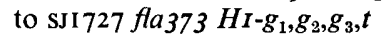

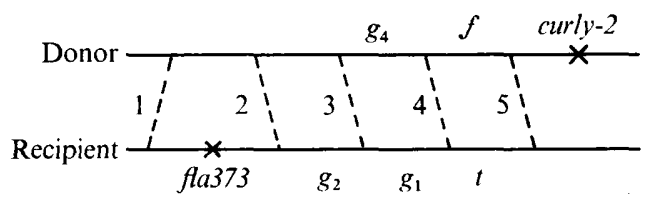

(B) Transduction from SJ $1892 H I-g_{3}, g_{4}, f$,curly-2 to SJI632 flaAII25I HI- $g_{1}, g_{2}, g_{4}, g_{5}, m$



$\begin{array}{cc}\begin{array}{c}\text { Factor composition } \\ \text { of recombinants }\end{array} & \begin{array}{c}\text { Crossover } \\ \text { regions }\end{array}\end{array}$

' $\mathrm{g}_{3}, \mathrm{~g}_{4}, \mathrm{f}$ '

' $\mathrm{g}_{1}, \mathrm{~g}_{2}, \mathrm{~g}_{3}, \mathrm{t}$ '

' $\mathrm{g}_{1}, \mathrm{~g}_{3}, \mathrm{t}$ '

' $\mathrm{g}_{3}, \mathrm{~g}_{4}, \mathrm{t}$ '

$I$ and 5

$I$ and 2

$\mathrm{I}$ and 3

$I$ and 4

Summing up the results of these two crosses and the previous report (Yamaguchi \& Iino, 1969), the following order is obtained:

$$
-f l a A I I-f l a A I-\overbrace{g_{2}-g_{1}-g_{4}-\left(g_{3}, g_{5}, f, m, t\right)-c u r l y-2}^{H I} \text { flaL-. }
$$

As curly-2 was allocated to segment 15 , these results show that all the antigenic specificitydetermining areas are distributed on segments 15 and/or 16. 


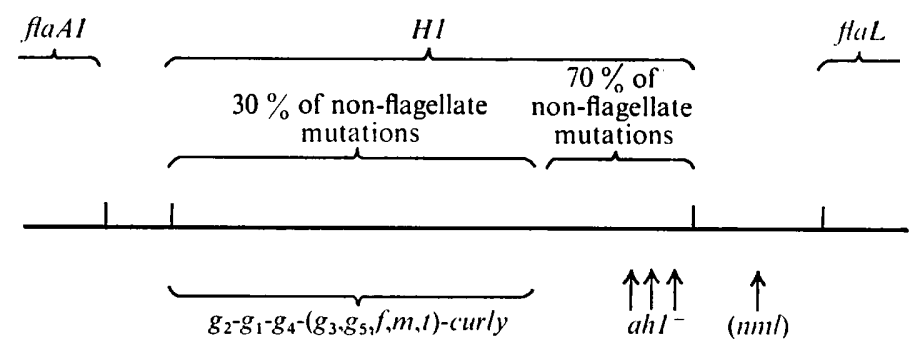

Fig. 4. Summarized map of $f a A I-H I-f l a L$ region.

\section{DISCUSSION}

The results obtained here are summarized in Fig. 4. As shown in the map, about $70 \%$ of non-flagellate $H I$ and all the $a H^{-}$mutational sites were located in the quarter of the $H I$ gene at its $f a L$ side, while curly mutational sites and antigenic specificity-determining areas were located in the remaining three quarters.

The $H I$ mutants studied in the present experiments are presumed to have defects in the synthesis of intact phase-I flagellin which can polymerize into flagellar filaments. It was demonstrated here that about $70 \%$ of non-flagellate mutational sites are concentrated in the $f a L$-side quarter of $H I$. Further, this region was shown to cover none of the antigenic specificity-determining area. These results imply that the region corresponds to a section of the polypeptide chain occupying an inner part of the tertiary structure of flagellin and playing an important role in the conformation and/or polymerization of the molecule. The antigenic specificity-determining areas, allocated on the three quarters of the $H I$ gene region at its flaAI side, may correspond to a part of the flagellin polypeptide exposed on the surface of flagellin molecule. The relatively rare detection of non-flagellate mutations in this region may mean that conformational alteration of flagellin caused by a mutational change in its surface is usually not so severe as to result in the loss of ability to polymerize. The above results and considerations conform with those of Parish, Wistar \& Ada (I969), who demonstrated by chemical and immunological analyses on fg-type flagellin that all the antigenic specificities reside in the central region of flagellin polypeptide, and thus inferred that this region forms the portion of a flagellar molecule exposed on the surface of a flagellar filament. In the present study, however, the presence of an antigenically-inert area to the $f l a A I$ side of $H I$ was not detected.

It has been reported that, not only in Salmonella but also in Bacillus subtilis, flagellarshape mutants are not accompanied by any change in serological alteration (Iino, 1962; Iino \& Mitani, 1967; Martinez et al. 1968). Therefore, the key region in flagellin in determining flagellar shape has been assumed to be distinct from those determining antigenic specificities. The present results confirm this assumption: a curly mutational site was separable from the antigenic specificity-determining areas in $H I$.

$H I$ and $a h I$ were initially defined as the phase-I antigen determinant and the activity controller of $H I$ adjacent to it, respectively (Lederberg \& Edwards, I953; Iino, I96I). Later, $H I$ was identified as the structural gene of phase-I flagellin, and $a h^{-}$was thought to be either an operator-negative state or nonsense chain-terminating mutations in $H I$ (Iino, I969). From the present experiments, it is now known that not all the HI region is responsible for determining the antigenic specificities of flagellin, and the same phenotype as $a h I^{-}$, i.e. defective phase-I flagella formation, can occur by a mutation in at least a 
quarter of $H I$ covering none of the antigenic specificity-determining areas. To avoid confusion between the symbols $H I$ and $a h I$, we propose to define $a h I$ hereafter as the operator region of $H I$, by analogy with the $a h 2-H 2-r h I$ operon (Fujita, Yamaguchi \& Iino, I973; Suzuki \& Iino, I973). By this definition, the mutations previously described as ' $a h^{-}{ }^{-}$' are not $a h^{-}$but nonsense $H I$ mutations. The genuine $a h I$ region is expected to be identified by isolation of mutants insensitive to the phase-I repressor.

The two-factor crosses showed the presence of intervals of some length between $H I$ and $f a A I$ and $H_{I}$ and $f l a L$. In particular, the length of the latter interval is comparable to that of the $H I$ gene. Stocker, McDonough \& Ambler (196I) demonstrated that $n m l$, a gene controlling the methylation of lysine residues of flagellin, is closely linked to the $H I$ gene. Recently, we have assigned the position of $\mathrm{nml}$ to the space between $\mathrm{HI}$ and flaL (unpublished).

\section{REFERENCES}

ENomoto, M. \& InNo, T. (1966). The comparison of normal and curly flagella in Salmonella abortusequi by two-dimensional separation of peptides. Japanese Journal of Genetics 4I, I3 I-I39.

Fujita, H., Yamaguchi, S. \& InNo, T. (1973). Studies on H-O variants in Salmonella in relation to phase variation. Journal of General Microbiology 76, $127-134$.

IINo, T. (1961). Genetic analysis of O-H variation in Salmonella. Japanese Journal of Genetics 36, 268-275.

InNo, T. (I962). Curly flagellar mutants in Salmonella. Journal of General Microbiology 27, 167-175.

IINo, T. (1969). Genetics and chemistry of bacterial flagella. Bacteriological Reviews 33, 454-475.

IINO, T. \& MitANI, M. (1966). Flagellar-shape mutants in Salmonella. Journal of General Microbiology 44, 27-40.

IINO, T. \& Mitani, M. (1967). A mutant of Salmonella possessing straight flagella. Journal of General Microbiology 49, 8I-88.

JoYs, T. M. \& STOCKER, B. A. D. (1969). Recombination in $H I$, the gene determining the flagellar antigen- $i$ of Salmonella typhimurium: mapping of $\mathrm{HI}_{\mathrm{I}}$ and fla mutations. Journal of General Microbiology 58, 267-275.

Lederberg, J. \& EdWARds, P. R. (1953). Serotypic recombination in Salmonella. Journal of Immunology $71,232-240$.

Martinez, R. T., Ichiki, A. T., Lundh, N. P. \& Tronick, S. R. (I968). A single amino acid substitution responsible for altered flagellar morphology. Journal of Molecular Biology 34, 559-564.

MeYnell, E. W. (1961). A phage, $\phi \chi$, which attacks motile bacteria. Journal of General Microbiology 25, 253-290.

PARISH, C. R., WISTAR, R., JUN. \& ADA, G. L. (I969). Cleavage of bacterial flagellin with cyanogen bromide: antigenic properties of the protein fragments. Biochemical Journal II3, 50I-506.

SASAKI, I. (I962). $\chi$-phage resistance in Salmonella having g-group antigens. Virus 12, 168-I76. (In Japanese.)

SUZUKI, H. \& IrNo, T. (1973). In vitro synthesis of phase-specific flagellin of Salmonella. Journal of Molecular Biology 81, 57-70.

Stocker, B. A. D., MCDonough, M. W. \& Ambler, R. P. (196I). A gene determining presence or absence of $\epsilon$ - $N$-methyl-lysine in Salmonella flagellar protein. Nature, London $\mathbf{1 8 9}, 556-558$.

YAMAGUCHI, S. (1968). Sensitivity of the $g$-complex antigenic Salmonella strains to M8, a host-range mutant of bacteriophage $\chi$. Journal of General Virology 2, 187-190.

YAMAGUCHI, S. \& IINO, T. (I969). Genetic determination of the antigenic specificity of the flagellar protein in Salmonella. Journal of General Microbiology 55, 59-74.

Yamaguchi, S., Iino, T., Horiguchi, T. \& Ohta, K. (1972). Genetic analysis of $f a$ and mot cistrons closely linked to $H_{I}$ in Salmonella abortusequi and its derivatives. Journal of General Microbiology 70, 59-75. 